\title{
The extent of processing of noise elements during selective encoding from visual displays*
}

\author{
CHARLES W. ERIKSEN and JAMES E. HOFFMAN \\ University of Illinois, Champaign, Illinois 61820
}

\begin{abstract}
Circular visual displays of 12 elements, consisting of $\mathbf{A}, \mathrm{H}, \mathbf{M}$, and $\mathrm{U}$, were presented to $\mathrm{S}$. She responded with a lever movement in one direction if a given letter designated by a bar indicator was a member of the set $A-U$ and in the opposite direction if it was from the other set. The principal experimental variables were the SOA by which the indicator preceded the display and whether the target letter was flanked by letters of the same or opposite set. The results indicated that the interference produced by noise letters is primarily on the response as opposed to the processing side. Also, there is a limit to the precision of selective attention in the visual field. The interference of opposite-set letters was inversely proportional to their distance from the target.
\end{abstract}

If a human $S$ is required to voice a target letter as quickly as possible from a multiletter display, his reaction time (RT), as might be expected, is faster if he has prior knowledge as to the location of the letter in the display. When this information is provided in the form of a line indicator designating the position, we have found that the decrease in RT becomes asymptotic when this indicator precedes the display by $200-300 \mathrm{msec}$ (Colegate, Hoffman, \& Eriksen, 1973). We have interpreted this in terms of the time required by $S$ to perceive and process the indicator information.

However, even when the indicator precedes the display by $300 \mathrm{msec}$, the number of noise letters or signals that are presented in the display along with the target letter influences RT. Time to voice the target letter remains slower, for example, if the display contains 11 noise letters rather than 7 .

Not only does the number of noise elements affect RT, but so does the nature of these noise elements and their spatial proximity to the target letter. Solid black disks increase RT appreciably less than the same number of letters, and the effect of both of these noise elements is enhanced if they lie within 1 deg of visual angle of the target letter (Eriksen \& Hoffman, 1972a).

In order to understand how $S$ can attend to or selectively encode one stimulus from a set of equally potent stimuli, it would be helpful if we knew the fate of the nonattended or noise stimuli. How much processing do they receive, and are those noise elements located in close proximity to the target processed further than those more remote? Does the effect due to number of noise elements and to their proximity to the target lie in the possibility that these elements are encoded and compete in terms of response with the target letter or does the competition lie in terms of feature analyzers and the encoding mechanism itself?

When the indicator occurs $350 \mathrm{msec}$ before the onset of the display containing target and noise, there is still

*This investigation was supported by U.S. Public Health Service Research Grant MH-1206 and U.S. Public Health Service Research Career Program Award K6-MH-22014. the effect of the noise on RT (Colegate, Hoffman, \& Eriksen, 1973). This would suggest that the noise elements are affecting the processing of the target rather than the perception of the indicator. One mode of action might be through response competition. If one of the noise signals should complete processing through to the point of encoding before the target letter itself is ready for encoding, it might lead to an incipient response incompatible with the response to the target letter.

Another mode of action might be through delaying the processing of the target letter due to competition with subprocessing units such as feature analyzers. In more general terms, the effect could be through the noise utilizing part of a limited supply of processing energy.

In the present experiment, we wished not only to elucidate more definitively the locus of the action of noise signals, but also to determine whether noise signals throughout the display contributed equally to the effect upon RT. Circular displays, consisting of 12 letters located in the clock positions, were used. The letters were drawn in various ways from the set of capital letters $A, H, M$, and $U$. As in our previous work, the target letter was indicated by a line external to the circumference of the circular arrangement of letters, with one end of the line designating the target position. The S's task was to move a response lever in one direction if it was an $\mathbf{H}$ or an $\mathbf{M}$ and in the opposite direction if the target was an $\mathrm{A}$ or a U. The experimental manipulations of greatest interest were the composition of the noise letters in the display, whether or not they were a member of the same response set as the target letter, and their spatial location in the display with reference to the target letter.

It was anticipated that if noise increased RT due to response competition, the maximal elevation in RT would occur when all the noise elements in the display consisted of letters of the opposite set from that of the target letter. On the other hand, when all the noise let ters consisted of letters from the same set as the target 
D1

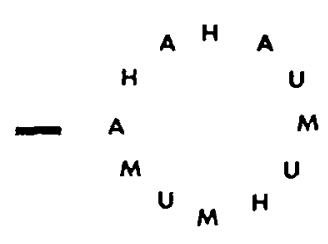

AD

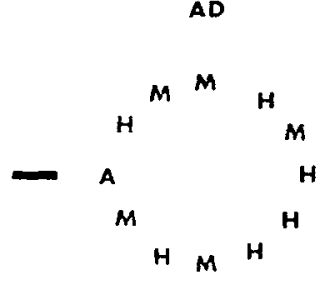

AD 54

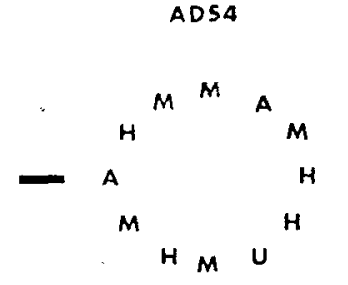

D2

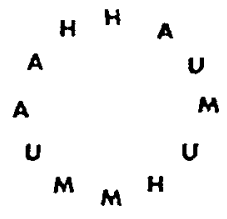

AS

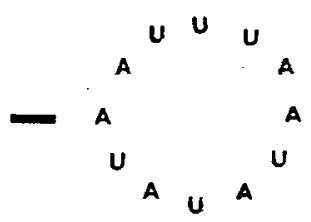

D 4

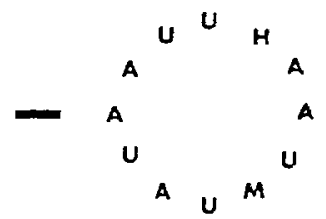

Fig. 1. Illustrations of representative display compositions for the six experimental conditions. Each condition is shown for the trial on which the target letter was an " $A$ " occurring in the 9 o'clock position.

letter, processing of one or more of these noise elements should not lead to an incompatible response. However, should noise contribute to RT by competing for processing units or for processing energy with the target letter. RT to displays of letters from a homogeneous set should lead to longer RTs than for a control condition where only the target letter appears in an otherwise blank display. It was further anticipated that these possible effects would vary as a function of where in the display noise letters of the opposite set were located with reference to the target letter. Thus, noise letters of the opposite set to that of the target would have their maximal interfering effect when they occupied display positions immediately adjacent to the target as opposed to positions further removed.

\section{EXPERIMENT I}

\section{Method}

\section{Subjects}

Four female students served as paid Ss, and all had normal or corrected-to-normal vision.

\section{Apparatus and Stimuli}

A Scientific Prototype Model GA three-fjeld tachistoscope

equipped with Sylvania F4T5/CWX fluorescent lamps was employed. Luminances in the three fields were maintained at $6 \mathrm{~mL}$, as measured with a Spectra Spot Photometer. A black fixation cross, $.6 \mathrm{deg}$ of visual angle, appeared in Field 1 . the adaptation field. which remained on except when field 3 containing the display was on. Field 2 contained a black line indicating one of the display elements for report. This indicator was $.5 \times .1 \mathrm{deg}$ of visual angle mounted on a white card. It was positioned so that it fell outside of the imaginary circle along which all display letters were arranged, and its nearest position was $.5 \mathrm{deg}$ of angle from the indicated letter. Further, it was positioned so that it would constitute an extension of an imaginary radius from the center of the display through the indicated position.

The $S$ initiated a trial by pressing a microswitch. which triggered the tachistoscope as well as a Hunter Model $1052 \mathrm{H}$ digital clock set to display RT in milliseconds. She responded by pushing a spring-loaded switch left or right with her right hand, which stopped the clock.

Display cards were constructed by placing capital letters, A, H, $U$, and $M$ (obtained from Paratipe No. 11316), subtending . $2 \mathrm{deg}$ of visual angle in height, on white vinyl cards. Letters were positioned in the 12 clock positions of an imaginary clock face, $2 \mathrm{deg}$ of visual angle in diam, appearing in the center of the viewing field. Six display cards were constructed for each of the 12 clock positions by leaving the indicated position blank and filling the remaining positions according to the rules that defined the six experimental conditions. Target-letter cards were constructed by placing each of the letters singly on clear plastic cards. Forty-eight of these cards were constructed, corresponding to each of the four target letters in each of the 12 positions. The target card and display card were placed in Field 3, resulting in a 12 -letter display.

\section{Procedure}

The four letters were divided into two sets: $A-U$ and $H-M$. Two of the Ss were instructed to push the switch Jeft if the target was an $A$ or a $U$ and right if it was an $H$ or an $M$. The other two Ss were given the opposite directions for each set. The six experimental conditions were defined in terms of the context of noise letters in which the target letter appeared. In Condition D-1, the display positions immediately adjacent to the target letter on each side were filled with the letters of the opposite set, the next positions were filled with letters from the same set, and the remaining display positions were filled randomly with the four possible letters. Thus, if the target letter was an $A$ or a $U$, the position on one side of the target letter would contain an $\mathrm{H}$ and the position on the other side an $\mathrm{M}$. In Condition D-2, the target letter was flanked on each side by a letter of the same set. Thus, if the target letter was an A, the display position on one side of the target would also contain an $A$ and on the other side a $U$. The second position on each side of the target letter, however, contained the letters from the opposite set. The rest of the display positions were then filled randomly from the four possible letters. In Condition D-4, the noise letters surrounding the target were all of the same set as the target, but four positions removed on each side of the target occurred the two letters from the opposite set. In the A-S condition, all 12 letters in the display were of the same set as the target, and in the A-D condition, all 11 noise letters in the display were of the opposite set. In the A-D-S-4 condition, all the noise letters were of the opposite set to the target except for the display positions four removed on each side of the target, which were occupied by members of the same set as the target. A final control condition, S-L, was employed in which only the target letter appeared in one of the 12 clock positions on an otherwise empty display.

Figure 1 illustrates a representative display for each of the experimental conditions for a trial where the target was the letter $A$ occurring in the 9 o'clock position.

The stimulus onset asynchrony (SOA) by which the indicator 
preceded the display was $0,50,150$, or 250 msec.

Each $\mathrm{S}$ served in two practice sessions followed by 12 experimental sessions. She was given eight practice trials on the first condition to be run each day. She was instructed to initiate a trial when the fixation cross appeared in good focus. At initiation, the adaptation field remained on with onset of Field 2, which contained the indicator. At SOAs of $0,50,150$, and $250 \mathrm{msec}$ after onset of Field 2, the adapting field terminated and was replaced by Field 3, which contained the 12-letter displays. Fields 2 and remained on together for $1 \mathrm{sec}$.

Each session consisted of four blocks of 28 trials each, one block under each of the four SOAs. Within a block, each of the seven conditions appeared four times in random order. Across sessions, each letter appeared equally often in each of the 12 clock positions for each conditions by SOA yielding 48 latency measures per $\mathrm{S}$ for each of the 28 conditions by SOAs.

Errors within conditions showed the same pattern as the RT data, with the most errors occurring in conditions with the longest RTs. Error rates within conditions ranged from $14 \%$ to $1 \%$, with an average of $6 \%$ across conditions and Ss. All error trials were mixed randomly into later blocks and rerun, so each data point is based on 48 correct latencies.

\section{EXPERIMENT II}

Upon completion of the above experiment, some uncertainty was found as to whether or not performance under the D-1 and D- 2 conditions eventually equaled the performance obtained under the no-noise or S-L condition when the indicator preceded the display by the longer SOAs. A partial replication of the above experiment was carried out, employing the D-1, D-2, and S- $\mathrm{L}$ conditions. In addition to SOAs of 0,50 , and $150 \mathrm{msec}$ by which the indicator preceded the display, an additional SOA of $350 \mathrm{msec}$ was studied for all three conditions. The data in this replication were gathered while the Ss participated in a different, but somewhat related, RT study. Three of the Ss were the same as had participated in Experiment $I$, and one new S (female) was recruited.

\section{Results}

In Fig. 2, the mean RT averaged over Ss is shown as a function of the SOA by which the indicator preceded the display for each of the seven experimental conditions in Experiment I. A three-way analysis of variance (conditions, SOAs, and Ss) was performed on these data. All main effects and interactions were significant at the .01 level or beyond, except for the Ss by Conditions interaction. A second analysis of variance, in which the S-L condition was eliminated, gave similar results, except that the Conditions by SOA interaction was no longer significant $[F(15,45)=1.76, p>.05]$.

As is seen in Fig. 2, there is little difference between Conditions A-D, D-1, and D-S-4. All three of these conditions have in common the presence of opposite-response noise letters in the positions immediately adjacent to the target letter. For Condition D-2, where same-response letters are in the immediately adjacent positions to the target and opposite-response letters are two positions removed. RT

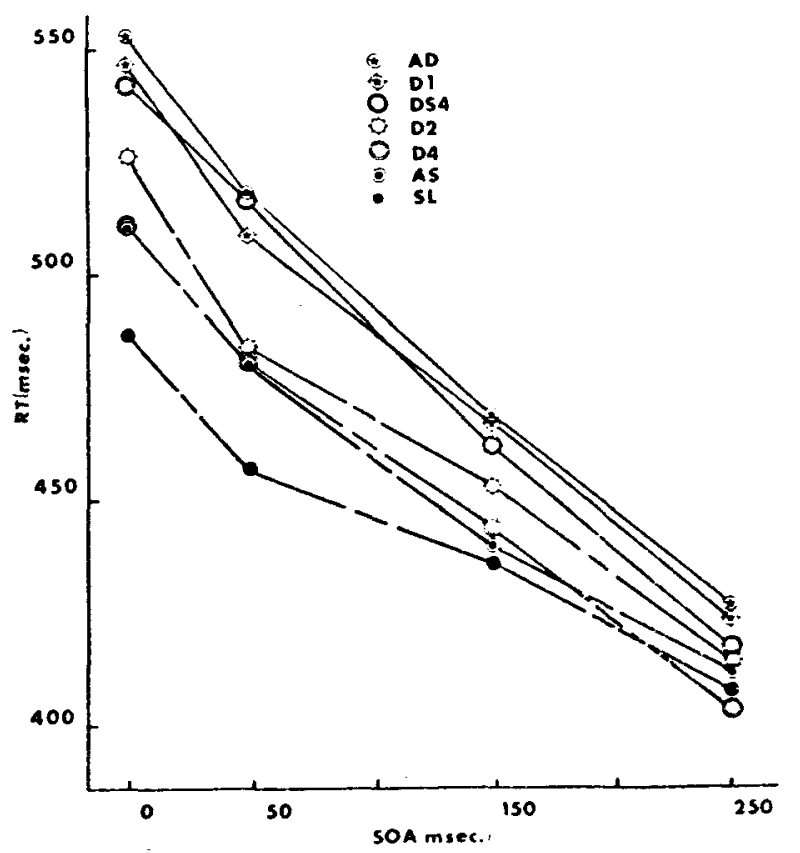

Fig. 2. Mean RT for each condition averaged over Ss as a function of the interval (in milliseconds) by which the indicator preceded the display.

is somewhat faster over the range of SOA values studied than for the above three conditions. D.2 is somewhat slower than Conditions A-S and D-4. In these latter two conditions, all noise letters were the same set as the target, except in D-4, where two opposite-response letters occurred in positions four removed on each side of the target. By the time the indicator has preceded the display by $250 \mathrm{msec}$, performance under these last two conditions is essentially the same as that obtained for Condition S-L, the single-letter display.

The shortest RTs are obtained for the S-L condition, where no noise letters were present, but even here there is a prominant effect for a leading indicator. RT decreases as the indicator precedes the display by intervals out to $250 \mathrm{msec}$. All conditions show this effect of the SOA of the indicator and the significant SOA by Condition interaction can be attributed to RT under all experimental conditions, tending to approach that of the S-L display as the indicator precedes the displays by increasing intervals.

While the A-S and D-4 conditions achieve the same RT level as the single-letter display at the longer SOA levels, the present data are unclear as to whether the effects of opposite-response noise letters close to the target would be overcome if the SOA of the indicator were long enough. Experiment II was carried out to provide further information on this point, and the SOA variable was extended to $350 \mathrm{msec}$. The results of this second experiment are contained in Fig. 3.

For all three conditions, D-1. D-2, and S-L, the results closely replicate those obtained in Experiment I. They also show that even when the indicator precedes the 


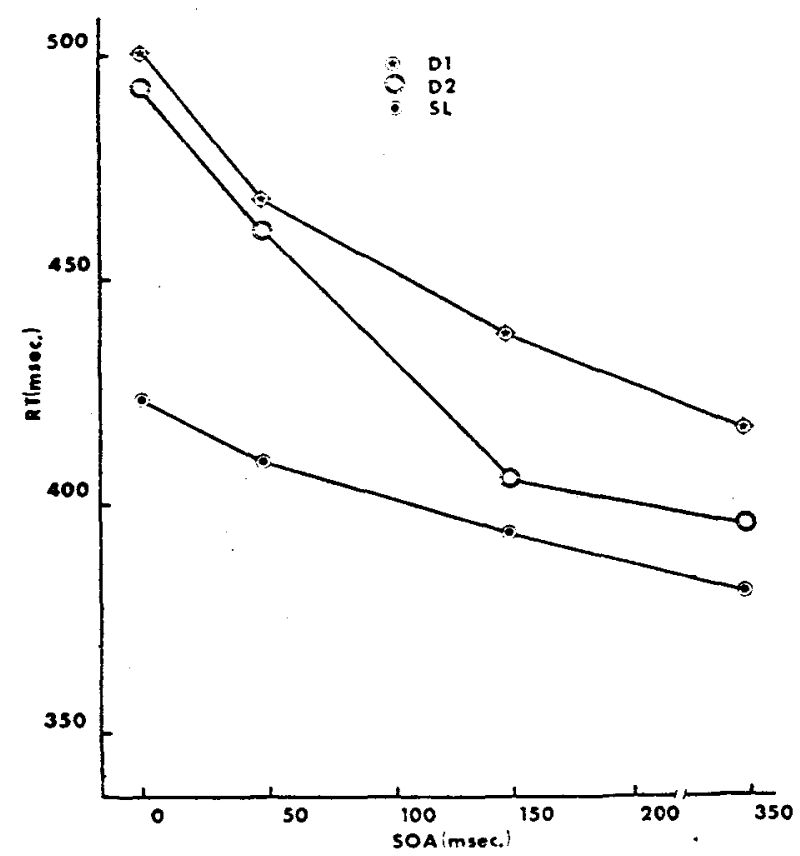

Fig. 3. Mean RT for the three replicated conditions in Experiment II as a function of the SOA by which the indicator preceded the display.

display by $350 \mathrm{msec}$, the effects of opposite-response noise letters immediately adjacent to the target letter are not overcome. RT at $350 \mathrm{msec}$ SOA is $33 \mathrm{msec}$ longer for the D-1 condition and $11 \mathrm{msec}$ longer for the D-2 condition than is obtained for the single-letter display. The analysis of variance supports these observations. All main effects and interactions were significant at or beyond the .01 level, except for Ss by Conditions.

\section{DISCUSSION}

There are three salient features in the above results that require discussion and an attempt at explanation. First, there is the effect of opposite- as opposed to same-response noise letters in close proximity to the target letter. Opposite-response noise letters result in slower RTs than do same-response letters at all values of SOA of the indicator.

Second, there is the decreasing difference between the single-letter condition and the other six experimental conditions as the indicator precedes the display by increasing SOAs. The RT differences between S-L and the other six conditions are maximal when the indicator occurs simultaneously with onset of the display, but by the time the indicator has preceded the display by $250 \mathrm{msec}, \mathrm{RT}$ s under the A-S and the D-4 conditions are essentially the same as that obtained with S-L. ${ }^{1}$ In the A-S condition, all the noise letters require the same response as the target, and in the D-4 condition, the letters in the three positions on each side of the target letter are of the same response set. For the four conditions where the displays contained opposite-response letters within two display positions of the target letter, there are still RT differences at the longest SOA values studied. However, the RT differences between these four conditions (A-D, D-1, D-2, and D-S-4) and the two other experimental conditions, A-S and D-4, remained essentially the same over all SOA values, as indicated by a nonsignificant Condition by SOA interaction when the data were analyzed excluding the S-L condition.

The third salient result is that the retarding effect upon RT of opposite-response letters is inversely proportional to their spatial separation from the target letter. RT is slowest when opposite-response noise letters occupy the immediately adjacent positions to the target. The effect is less if they are removed two positions, and if four positions removed, the effect of opposite-response noise is no different from that obtained with same-response noise letters.

The differential effect of opposite- vs same-response noise letter was an anticipated effect. As was pointed out in the introduction, there are several nonexclusive ways that noise letters could impede RT to the target letter. On the processing side, they could compete for subprocessing units such as feature analyzers. Or, if the encoding mechanism itself is serial in nature, they could arrive at an encoding stage before the target letter, thus. delaying encoding of the target. If encoding or processing is essentially multichannel, at least to a limited extent, the noise letters could be retarding RT due to eliciting or tending to elicit competing or incompatible responses.

The present results tend to localize the effect on the response side. When the indicator precedes the display by $250 \mathrm{msec}$, noise letters, if they elicit the same response as the target letter, produce RTs that are no longer than those obtained to the no-noise display (S-L). On the other hand, if the noise letters elicit the opposite response and are within one or two positions of the target, RTs remain longer than for the no-noise display, even if the indicator has preceded the display by $350 \mathrm{msec}$.

At this point, the interaction between conditions and SOA must be considered. In prior work (Eriksen \& Hoffman, 1972b; Colegate, Hoffman, \& Eriksen, 1973), we have ascribed the effect of a leading indicator as due to the provision of time for the $S$ to locate and process the indicator information. We have found that the RT-SOA function becomes asymptotic when the indicator precedes the display by between 200 and $300 \mathrm{msec}$. Further, the asymptotic RT value is dependent upon the number of noise elements in the display, with larger displays yielding longer asymptotic RTs. In these prior studies, the noise elements in the display led to competing responses with the target letter, but there, as in the present experiments, the greatest differences in RTs were obtained when the display and indicator occurred simultaneously.

If we assume that between 200 and $300 \mathrm{msec}$ is 
required to encompass the latencies in processing the indicator information, we can attribute the greater differences between conditions at zero SOA in the experiments as due to two components. First, the number of noise signals interferes with the location of the indicator and the processing of the information; and second, the noise letters tend to elicit competing responses. When the display and indicator occur simultaneously, the speed with which the indicator is located and processed depends upon the complexity of the visual field. The more noise signals, the longer the time required to locate the indicator and process it. Once the indicator has been processed, the RT to the target letter depends upon whether the noise signals in close spatial proximity elicit the same or opposite response tendencies.

This explanation says that the difference in RT between same- and opposite-response noise letters is essentially the same over the SOA range, but the decreasing difference between these conditions and the no-noise condition as SOA increases is attributable to the decreasing effect of noise elements on location and processing of the indicator information. This is consistent with the significant interaction between conditions and SOA when the S-L condition is included in the analysis and the lack of a significant interaction when the S-L condition is excluded.

How does the noise interfere with the location and processing of the indicator information? One possibility is that while the indicator is being detected and processed, the visual perceptual system is also randomly processing some of the elements or letters in the display. In other words, before the $\mathrm{S}$ knows which letter to report, he may process one of the noise letters, and if this leads to an incompatible response, RT to the target letter will be delayed. This possibility would seem to be excludable on the basis of the present data.

If processing of one or more randomly selected letters from the display was occurring while the indicator information was being processed, we would expect a difference in RT between Conditions D-4 and A-S and between D-S-4 and A-D. In the D4 condition, all display letters require the same response as the target except for two letters four positions removed from the target. In D-S 4 , all letters in the display are of opposite response to the target except for two letters four positions removed. If the $S$ was randomly processing some of the letters of the display while processing the indicator, one would expect that on one-sixth of the trials the opposite response letters in the D4 condition would be those processed. Over a large number of trials, the sampling of opposite-response letters on one-sixth of the trials should lead to a systematic difference between the D-4 condition and A-S, where all letters are from the same response set. Similarly, on one-sixth of the trials in the D-S-4 condition the letter sampled would be from the same response set leading to shorter RTs than for the A-D condition. Such differences are not confirmed by the data. Performance under D-4 is essentially the same as that obtained when all noise letters are the same response set and performance under the D-S-4 is essentially the same as when all noise letters are of the opposite response set.

A different possibility is that the number of noise letters increases the difficulty of locating or discriminating the indicator. Estes (1972) has shown that search time for targets in visual displays increases as the number of noise elements in the displays increases and that this effect of noise elements decreases as the dissimilarity between the target and noise elements is increased. In the present experiment, having the indicator precede the display by increasing intervals may have served to make the indicator more discriminable from the noise. Thus, its location time decreases.

We recognize, though, that this is essentially begging the question, since we do not understand the processing mechanisms that underlie differences in discriminability between stimuli within and between modalities. To say that RT is faster under one condition than under another because the target is more discriminable under the first condition is essentially circular. Research on information processing must soon address itself to the processing mechanisms that underlie discriminability. For now, we may suggest that visual perception is basically object oriented and, when a new stimulus appears in the visual field, a certain level of gross processing and structuring of the stimulation is a prerequisite to more careful selective processing of detailed features of the stimulation. The more complex the stimulation, the longer the initial time required in the structuring before selective feature processing can occur.

The above discussion of the effects of same- as opposed to opposite-response noise has been restricted to the cases where the noise is in close spatial proximity to the target letter. The present results show a quite clear ordering in terms of RTs, depending upon whether the opposite-response noise letters are in the immediately adjacent positions to the target (D-1) two positions removed (D-2) or four positions removed (D-4). This effect is still apparent even when 250-350 msec have been available for the location and processing of the indicator information.

This result is consistent with the previous finding of Eriksen and Hoffman (1972a), where physical proximity of noise elements to the target letter was found to be quite critical within a degree of visual angle. Beyond $1 \mathrm{deg}$, differences in spacing had little or no effect. In discussing this previous result, we suggested that James's phenomenological characterization of attention as having a focus, margin, and fringe might have a measurable extent in the visual field. In terms of both the present and previous results, the focus would appear to be approximately $1 \mathrm{deg}$ of visual angle, with the margin extending slightly more (corresponding to the D-2 results in the present experiment) and the rest of the 
field constituting the fringe. The relationship of this characterization to the distribution of acuity across the retina is quite obvious. In the case of the attentional field, instead of acuity, the distribution is in terms of the level of information extraction. Thus, the focus is characterized by detuiled information extraction rather than high sensory resolving power. In normal vision, the fovea and the focus of attention would normally coincide, since we tend to fixate that which we are attending. Under special circumstances, such as experimental conditions with tachistoscopic exposures, the two need not coincide, al though the area of the retina upon which the stimuli fall will limit the level of detail that is available to some central attentional mechanism.

The finding that opposite response noise elements within $1 \mathrm{deg}$ of visual angle of the target letter continue to slow RT even when the indicator has preceded the display by $250-350 \mathrm{msec}$ suggests that there is a limit on the precision with which detail can be selectively encoded or attended to in the visual field. This is consistent with the idea of an attentional focus, but extends the concept further in that it says there is an irreducible minimum size of this focus. The results we have obtained so far suggest that this minimum focus field size is $1 \mathrm{deg}$ of visual angle or less, as reflected in the visual field. Within this focus field, there seems to be a lack of precision in determining the order of information extraction. Even when $500 \mathrm{msec}$ have been allowed (Colgate, Hoffman, \& Eriksen, 1973), selective encoding of the target is still impaired by adjacent noise elements. Whether this impairment occurs due to a parallel processing of all information within the focus or a serial, but essentially random, extraction of information or detail in the focus cannot be determined from present data.

\section{REFERENCES}

Colegate. R. L.. Hoffman, J. E.. \& Eriksen. C. W. Selective encoding from multielement visual displays. Perception \& Psychophysics, 1973, in press.

Eriksen, C. W., \& Hoffman, J. E. Temporal and spatial characteristics of selective encoding from visual displays. Perception \& Psychophysics. 1972a, 12, 201-204.

Eriksen, C. W., \& Hoffman. J. E. Some characteristics of selective attention in visual perception determined by vocal reaction time. Perception \& Psychophysics, 1972b, 11. 169-171.

Eriksen, C. W. \& Lappin, J. S. Internal perceptual system noise and redundancy in simultaneous inputs in form identification. Psychonomic Science, 1965, 2, 351-352.

Estes, W. K. Interactions of signal and background variables in visual processing. Perception \& Psychophysics, 1972, 12, 278-285.

Hintzman, D. L., Carre, F. A., Eskridge, V. L., Owens, A. M., Shaff, S. S., \& Sparks, M. E. "Stroop" effect: Input or output phenomenon? Journal of Experimental Psychology, 1972, 95, 458-459.

\section{NOTE}

1. A consulting editor has pointed out that there is some evidence that would lead to the expectation that RT to the A-S. condition would be faster than to the S-L condition. Hintzman, Carre, Eskridge, Owens, Shaff, and Sparks (1972) found that color words printed in the corresponding color facilitated color-naming responses. Also, Eriksen and Lappin (1965) obtained increased identification accuracy for tachistoscopically presented letters when the letter was replicated two to six times in a single exposure.

(Received for publication November 15. 1972; accepted March 13, 1973.) 